\title{
Reuse of structural domain-domain interactions in protein networks
}

\author{
Benjamin Schuster-Böckler* and Alex Bateman
}

\author{
Address: Wellcome Trust Sanger Institute, Wellcome Trust Genome Campus, Hinxton, UK \\ Email: Benjamin Schuster-Böckler* - bsb@sanger.ac.uk; Alex Bateman - agb@sanger.ac.uk \\ * Corresponding author
}

Published: 18 July 2007

BMC Bioinformatics 2007, 8:259 doi:10.1186/147/-2105-8-259

This article is available from: http://www.biomedcentral.com//47/-2/05/8/259

(C) 2007 Schuster-Böckler and Bateman; licensee BioMed Central Ltd.

This is an Open Access article distributed under the terms of the Creative Commons Attribution License (http://creativecommons.org/licenses/by/2.0), which permits unrestricted use, distribution, and reproduction in any medium, provided the original work is properly cited.
Received: 23 February 2007

Accepted: 18 July 2007

\begin{abstract}
Background: Protein interactions are thought to be largely mediated by interactions between structural domains. Databases such as iPfam relate interactions in protein structures to known domain families. Here, we investigate how the domain interactions from the iPfam database are distributed in protein interactions taken from the HPRD, MPact, BioGRID, DIP and IntAct databases.

Results: We find that known structural domain interactions can only explain a subset of 4-19\% of the available protein interactions, nevertheless this fraction is still significantly bigger than expected by chance. There is a correlation between the frequency of a domain interaction and the connectivity of the proteins it occurs in. Furthermore, a large proportion of protein interactions can be attributed to a small number of domain interactions. We conclude that many, but not all, domain interactions constitute reusable modules of molecular recognition. A substantial proportion of domain interactions are conserved between E. coli, S. cerevisiae and H. sapiens. These domains are related to essential cellular functions, suggesting that many domain interactions were already present in the last universal common ancestor.

Conclusion: Our results support the concept of domain interactions as reusable, conserved building blocks of protein interactions, but also highlight the limitations currently imposed by the small number of available protein structures.
\end{abstract}

\section{Background}

One way to understand a protein's function is to look at its composition of conserved domains. Such families of related sequence regions, collected in the Pfam database [1], usually constitute structurally and functionally conserved modules. It is assumed that binding interfaces, too, are conserved evolutionary modules that are reused between proteins of different functions and retained during evolution $[2,3]$.
Therefore, domain-domain interactions are often regarded as the currency of protein-protein interactions. Based on this assumption, $\mathrm{Ng}$ et al. described an approach to predict domain-domain interactions using literature curation, evolutionary history and the distribution of domains in protein interactions [4]. Wuchty et al. compared the relationship between this set of predicted interacting domain pairs to the domain coocurrence network [5]. More recently, other groups have come up with sophisticated statistical methods to estimate putatively interacting domain pairs, based on the assumption of 
domain reusability [6-10]. However, none of these approaches offers structural evidence that the predicted domain pairs are able to form an interaction.

For complexes with known structure, it has been shown that domains can mediate interactions $[11,12]$. Such interactions between pairs of domains are stored in the $i$ Pfam database [13]. The structural evidence lends strong support to the inferred domain pair, resulting in a high confidence set of domain pairs.

Unfortunately, the selection of complexes in the PDB database of protein structures [14] is rather small and biased [15]. There is often only a single structure that shows a certain protein pair to interact, while other complexes like haemoglobin have been crystalized dozens of times. This makes it difficult to assess whether some domain pairs act as reusable modules in protein interactions from PDB data alone.

High-throughput experiments [16-18] and extensive literature curation efforts [19] have yielded large databases of protein interactions [20-24]. Despite the continuing growth of protein interaction databases, even the best studied protein interaction network of $S$. cerevisiae is thought to be incomplete and inaccurate [25-27]. Given that this network already comprises around 60000 interactions, questions arise as to how such networks have evolved and how they are organised. Furthermore, methods for assessing the quality of high-throughput experimental results are in high demand due to the error prone nature of the methods used.

In this study, we investigate how pairs of protein families taken from $i$ Pfam are distributed in experimental protein interactions from five major model species. This allows us to address a number of questions: what proportion of each organism's protein interaction network, its interactome, can be attributed to a known domain-domain interaction? How conserved are domain-domain pairs between species, and how many interacting domain pairs are still unknown?

\section{Results \\ iPfam domain pairs are overrepresented in experimental protein interactions}

We analysed the distribution of Pfam families known to interact from a PDB structure (iPfam domain pairs) in experimentally derived protein interactions (experimental interactions). The experimental interactions were filtered to only include interactions with exactly two partners (see Methods). The fraction of experimental interactions that contain at least one $i$ Pfam domain pair is referred to as the iPfam coverage. Accordingly, the fraction of experimental interactions that contains any pair of Pfam domains (excluding the $i$ Pfam domain pairs) is called the Pfam coverage.

Figure 1 shows the Pfam and $i$ Pfam coverage for the analysed species as a column chart. The number of resolved protein interactions varies greatly between species, as does the size of the underlying proteome (see Table 1). The Pfam coverage, coloured red in Figure 1, lies between $49.46 \%$ and $66.73 \%$. Given that $74 \%$ of all UniProt proteins contain at least one Pfam match, this is not by itself surprising. The iPfam coverage, shown in blue, is much smaller, ranging from $2.92 \%$ in D. melanogaster to $19.02 \%$ in $H$. sapiens. In $S$. cerevisiae the species with the most comprehensively studied interactome, the $i$ Pfam coverage is $4.47 \%$.

The relatively low $i$ Pfam coverage is by itself a disappointing finding. However, the fact that only a small fraction of protein interactions contain known domain pairs could be a result of the scarcity of available structures of protein complexes. Therefore, we asked whether the observed $i$ Pfam coverage is larger than would be expected by chance. To test this, we created 1000 random networks per species using the algorithm described in Methods. We then calculated the $i$ Pfam coverage on the protein interactions in each randomised network. Mean and standard deviations of the randomisation experiments are shown in Table 1. No P value (see Methods) was greater than $1.84 \cdot 10^{-06}$. This proves that the observed $i$ Pfam coverage is significantly higher than expected and $i$ Pfam domain pairs are enriched in real experimental protein interactions.

\section{Few iPfam domain pairs are responsible for a majority of the coverage}

To understand why $i$ Pfam domain pairs occur more often in experimental interactions than expected by chance, we analysed the two largest data sets, $S$. cerevisiae and $H$. sapiens in more detail. In the following paragraph, we will call the experimental interactions that contain an iPfam domain pair the covered experimental interactions. In Figure 2 , we compare the distribution of $i$ Pfam domain pairs on the number of experimental interactions for E. coli, S. cerevisiae and $H$. sapiens. This plot reflects how many $i$ Pfam domain pairs cover how many experimental interactions. Domain pairs that cluster to the left of the plot can be called specific domain pairs, as they only occur in very few covered experimental interactions. Conversely, domain pairs that cluster to the right of the plot occur in a large number of different covered experimental interactions and can be called promiscuous domain pairs.

All three distributions in Figure 2 resemble a power law distribution, according to the good fit of log-linear functions $(\log (f(x))=k \log x+\log a)$ shown as dotted lines. 


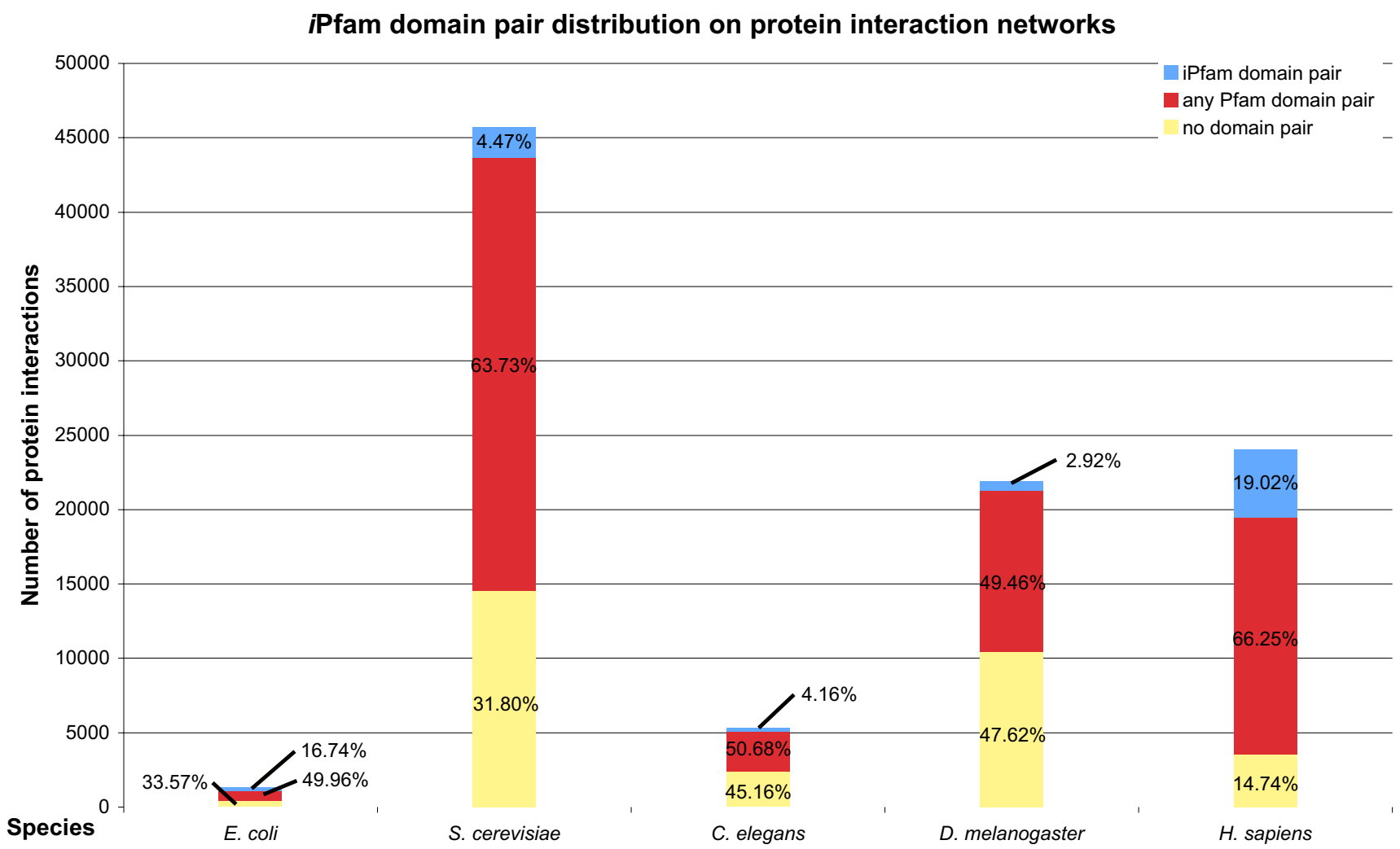

Figure I

Comparison of coverage of iPfam domain pairs on protein interactions. For each species, the height of the column reflects the number of known protein-protein interactions in the data set. The columns are split according to the proportion of interactions that contain an iPfam domain pair (blue), that contain any other Pfam domains on both proteins (red), and those that contain no Pfam domain pair (yellow).

The slopes $k$ of the H. sapiens and S. cerevisiae distributions are very similar (-1.53 and -1.60 , respectively), while $E$. coli has a markedly smaller slope $(-2.78)$. This suggests that the ratio of specific to promiscuous $i$ Pfam domain pairs is very similar in S. cerevisiae and H. sapiens, whereas E. coli features fewer multiply reoccurring $i$ Pfam domain pairs.
The power law distribution of $i$ Pfam frequencies implies that the majority of covered protein interactions can be attributed to a minority of $i$ Pfam domain pairs. $51.7 \%$ of the iPfam domain pairs in S. cerevisiae and $45.3 \%$ in $H$. sapiens are seen in just one experimental interaction. Conversely, $92.4 \%$ of $H$. sapiens and $85.4 \%$ of S. cerevisiae covered experimental interactions contain an $i$ Pfam domain pair that occurs more than once. Even more, half of the

Table I: iPfam domain pair coverage on protein interactions

\begin{tabular}{|c|c|c|c|c|c|c|}
\hline Species & $\begin{array}{l}\text { Proteins in } \\
\text { proteome }\end{array}$ & $\begin{array}{l}\text { \% proteome in } \\
\text { interaction set }\end{array}$ & $\begin{array}{l}\text { Protein pairs in } \\
\text { interaction set }\end{array}$ & $\begin{array}{l}\text { Protein pairs with } \\
\text { iPfam domain pair }\end{array}$ & $\begin{array}{l}\text { Protein pairs with } \\
\text { iPfam domain pair } \\
\text { (randomised mean) }\end{array}$ & $\begin{array}{l}\text { Standard } \\
\text { deviation }\end{array}$ \\
\hline E. coli & 4314 & $26.96 \%$ & $|28|$ & 211 & 178 & 7.12 \\
\hline S. cerevisiae & 5780 & $92.72 \%$ & 45707 & 2045 & 528 & 57.49 \\
\hline C. elegans & 22437 & $13.47 \%$ & 5310 & 221 & 76 & 9.90 \\
\hline D. melanogaster & 16251 & $43.22 \%$ & 21921 & 641 & 195 & 21.79 \\
\hline H. sapiens & 38213 & $21.40 \%$ & 24065 & 4577 & 1373 & 116.86 \\
\hline
\end{tabular}

For each species, we list the size of the proteome as defined in Integr8 and the fraction of this proteome that is represented in the protein interaction sets, followed by the total number of binary protein interactions and the fraction of those that contain an iPfam domain pair. The last two columns show the results of the network shuffling experiments. 


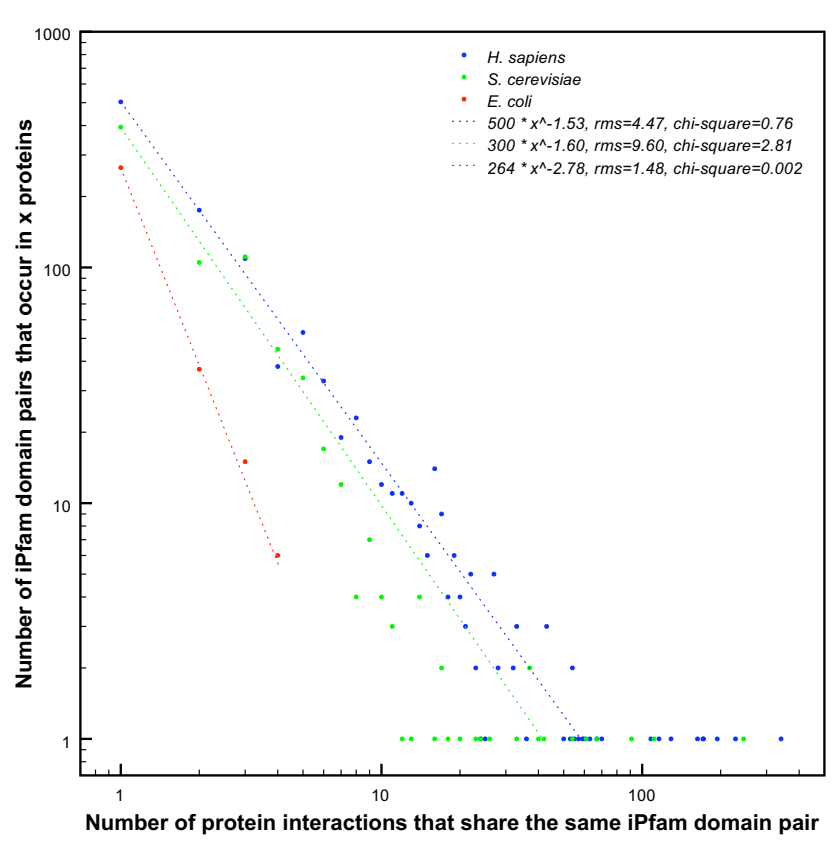

\section{Figure 2}

Frequencies of iPfam domain pairs in E. coli, S. cerevisiae and $H$. sapiens protein interactions. Each point in this graph represents a set of protein interactions. The abscissa reflects the number of interactions in each set that contain the same iPfam domain pair. The ordinate shows the number of distinct such sets, each defined by a different iPfam domain pair. In both $H$. sapiens (blue) and S. cerevisiae (green) a small number of iPfam domain pairs covers a large fraction of the interactome, whereas in E. coli, no iPfam domain occurs in more than 4 experimental interactions at a time. Dotted lines denote fitted monomial functions, showing that the distributions follow a power law.

covered experimental interactions in $H$. sapiens contain an $i$ Pfam domain pair that occurs in more than 16 different experimental interactions ( 5 for S. cerevisiae).

\section{Degree distribution and iPfam domain pair frequency are correlated}

We reasoned that if there are $i$ Pfam domain pairs that act as reusable modules in protein interactions, then highly connected proteins should also be more likely to contain promiscuous $i$ Pfam domain pairs and vice-versa.

For each node (i.e. protein) in the filtered $H$. sapiens and $S$. cerevisiae protein interaction network, we calculated its degree, defined as the number of adjacent edges (i.e. interactions). At the same time, we counted the number of $i$ Pfam domain pairs on the adjacent edges. In Figure 3, we plot the mean number of $i$ Pfam domain pairs relative to the degree of the node.

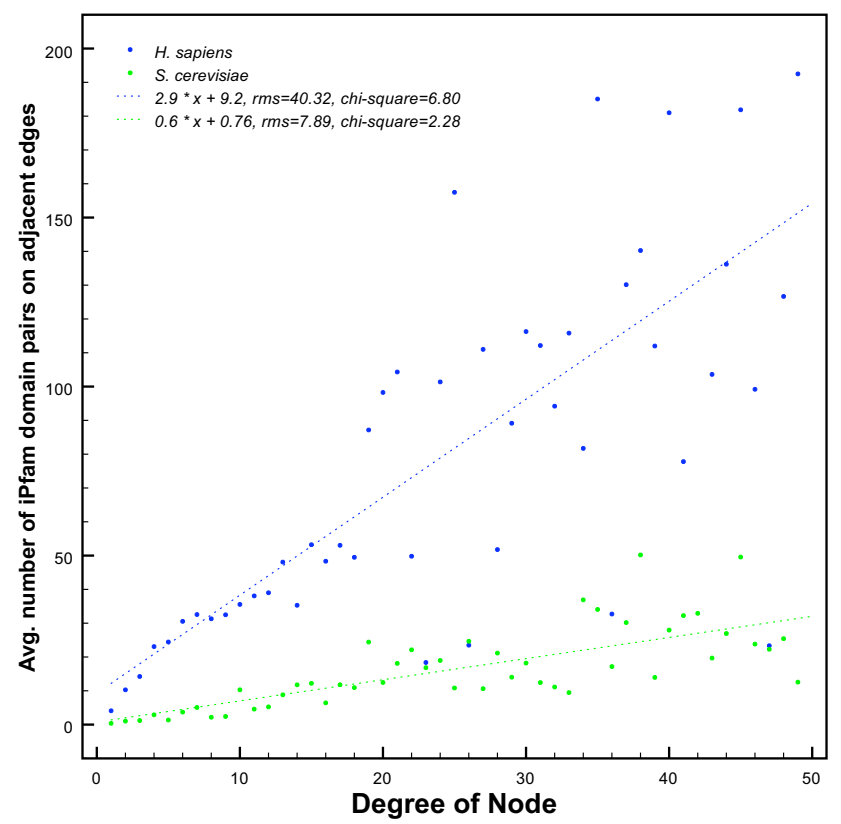

Figure 3

Average frequency of iPfam domain pairs relative to degree of node. Each point represents a protein in the interaction networks of $H$. sapiens (blue) and $S$. cerevisiae (green). For each protein, we calculate the degree, defined as the number of interactions the protein is involved in. On the $y$-axis, we show the average number of iPfam domain pairs in edges adjacent to proteins of degree $x$. We calculated a Spearman correlation of 0.68 and $0.7 \mathrm{I}$, for $\mathrm{H}$. sapiens and $\mathrm{S}$. cerevisiae. The correlation is outlined by dotted lines.

We find that for proteins from a degree of 1 to 50 , there is strong correlation in both $H$. sapiens and $S$. cerevisiae (Spearman correlation coefficients of 0.68 and 0.71 , respectively) between degree and number of $i$ Pfam domain pairs on adjacent edges. For the $1.2 \%$ of proteins in $H$. sapiens and $6.4 \%$ in S. cerevisiae which have a degree higher than 50 , the correlation gradually diminishes.

\section{Promiscuous domain pairs}

Additional file 1 contains a list of all $i$ Pfam domain pairs and their frequencies in the experimental protein interactions, while Additional file 4 lists the frequencies of the single domains. Interactions between protein kinase domains (Pkinase, Pfam acc. PF00069 and Pkinase_Tyr, Pfam acc. PF07714) are the most frequent $i$ Pfam domain pairs, as well as interactions involving recognition domains such as SH2 or SH3. In S. cerevisiae, the Proteasome family (Pfam acc. PF00227, a family of peptidases) and WD40 (Pfam acc. PF00400, a repeat involved in multimer assembly) are also amongst the five most frequent $i$ Pfam domain pairs. As expected, more frequent domains are also more likely to be found as pairs in interacting pro- 
teins. It should be noted however that in the PDB structures, some of the observed domain pairs (Pkinase_Tyr $\leftrightarrow$ SH3_1, Pkinase_C $\leftrightarrow$ Pkinase and others) are only seen to interact within one protein (intrachain interactions) as opposed to interactions between two distinct proteins (interchain interaction). The table in Additional file 5 lists the number of PDB structures for each $i$ Pfam domain pair, distinguishing between intrachain and interchain interactions. Looking for example at the covered experimental interactions in $H$. sapiens(Additional file 1 ), only 8 out of the 100 most frequent iPfam domain pairs are seen in intrachain interactions exclusively, while 61 are exclusive to interchain interactions and 31 are seen in both.

A possible explanation for the occurrence of purely intrachain iPfam domain pairs in the covered experimental interactions is that they frequently cooccur together on the same protein with other iPfam domain pairs. A list of all combinations of iPfam domains (the domain architecture) on interacting proteins is given in Additional file 2. It reveals that certain iPfam domains such as SH2, SH3_1 or Pkinase_tyr frequently occur in the same architecture. Without further experiments, we cannot assign the correct interacting domains with certainty.

This highlights a basic assumption of this study that could be a source of error. We assume that interacting proteins that contain an iPfam domain pair interact through these domains. This, of course, is not necessarily the case. Although it has been shown that sequence similarity is linked to the mode of interaction [28], not every protein interaction that contains an $i$ Pfam domain pair is necessarily mediated by exactly this domain pair. To gain a rough estimate of the false positive rate due to this assumption, we counted how many protein pairs in the PDB contain an iPfam domain pair that does not mediate an interaction in one complex structure but does so in another. 3671 out of a total of 5380 interacting protein pairs from the PDB contain an $i$ Pfam domain pair that does not interact in one complex structure but does so in another. This means that for more than $32 \%$ of the protein interactions in the PDB, the iPfam domain pair assignment is correct. For the remaining 68\%, the $i$ Pfam domain pair assignments are wrong in one case but correct in another. The real false positive rate is likely to be smaller, because some iPfam domain pairs might still independently mediate an interaction with a different, possibly unknown, partner protein.

iPfam domain pairs are enriched in S. cerevisiae complexes We tested whether $i$ Pfam domain pairs are enriched in known protein complexes from $S$. cerevisiae. This is interesting firstly because domain-domain interactions are thought to be more common in obligate interactions. Secondly, the described modularity of known S. cerevisiae complexes lends support to the assumption that the underlying $i$ Pfam domain pairs are modular. In fact, we find a two-fold enrichment for $i$ Pfam domain pairs in the complexes described by Gavin et al. [29]. From the 294 binary protein interactions in this data set, 24 contained an $i$ Pfam domain pair, which corresponds to a coverage of $8.16 \%$ (P value $\left.2.7 \cdot 10^{-47}\right)$.

We also analysed the full dataset of protein complexes. From 491 complexes described by Gavin et al., 157 contained at least one pair of proteins with an iPfam domain pair $(31.9 \%)$. In total we found 617 pairs of proteins that contained an $i$ Pfam domain pair. Interestingly, we find that the distribution of $i$ Pfam domain pairs on complexes is uneven. When we drew 617 protein pairs randomly from all possible protein pairs in the complexes, we covered 192 complexes on average, with a standard deviation of 7.22. The probability of covering only 157 complexes is just $6.24 \cdot 10^{-07}$. Thus, some complexes contain a greater number of $i$ Pfam domain pairs, while other complexes do not contain any at all. This suggests that some sets of domain pairs are specific to certain complexes or pathways. Typical examples are the RNA polymerase II complex (IntAct id: EBI-815049) or the U1 snRNP complex which contain numerous $i$ Pfam domain pairs that are specific to these complexes.

\section{iPfam domain pairs are conserved between species}

Within the 3 to $19 \%$ of experimental interactions covered by $i$ Pfam, we analysed the conservation of $i$ Pfam domain pairs between species. We call an iPfam domain pair conserved when the same pair is observed in experimental interactions of two different species. The matrix in Table 2 shows the pair-wise conservation of $i$ Pfam domain pairs. For each species, a maximum of $40 \%$ to $90 \%$ of $i$ Pfam domain pairs can also be found in another species, although not all overlaps are as large.

Figure 4 shows a Venn diagram of the mutual overlaps between the two eukaryotes $S$. cerevisiae and $H$. sapiens and the prokaryote E. coli. While the eukaryotes share 524 domain pairs, only 158 iPfam domain pairs are shared between $S$. cerevisiae and E. coli, and only 135 between $E$. coli and $H$. sapiens. Remarkably, 53\% of the observed $i$ Pfam domain pairs in E. coli are also observed in one of the two eukaryotes, and 107 Pfam domain pairs are even conserved amongst all three species. The $i$ Pfam domains in these pairs are related to housekeeping activities such as translation, replication or ATP synthesis. Additional file 3 contains a list of the conserved $i$ Pfam domain pairs.

We also compared the iPfam domain pair frequencies between $H$. sapiens and $S$. cerevisiae directly. We derive a Spearman correlation coefficient of 0.50 for the frequencies of all $524 \mathrm{iPfam}$ domain pairs that are conserved 
Table 2: Matrix of mutual shared iPfam domain pairs

\begin{tabular}{|c|c|c|c|c|c|c|}
\hline & E. coli & S. cerevisiae & C. elegans & D. melanogaster & H. sapiens & iPfam domain pairs in total \\
\hline E. coli & & 158 & 35 & 30 & 135 & 347 \\
\hline S. cerevisiae & & & 129 & 164 & 524 & 835 \\
\hline C. elegans & & & & 102 & 172 & 197 \\
\hline D. melanogaster & & & & & 241 & 266 \\
\hline H. sapiens & & & & & & $|22|$ \\
\hline
\end{tabular}

The Table shows the number of co-occurences of iPfam domain pairs between two species. The right-most column lists the total number of unique iPfam pairs found in each species' experimental interactions.

between $S$. cerevisiae and $H$. sapiens. To test whether the correlation is an artefact of the distribution of the values, we recalculated the correlation 1000 times, each time shuffling one distribution randomly. From these random results, we derive a P value of $3.6 \cdot 10^{-30}$ that the observed correlation is random. This suggests that $i$ Pfam domain pairs with a large number of occurrences in one species tend also to be more frequent in the other.

\section{Predicting the total number of iPfam domain pairs in nature}

Our analysis allow us to estimate how many $i$ Pfam domain pairs would eventually cover all protein interactions. This corresponds to the predictions made by Aloy and Russel [2]. Similar to their approach, we make a linear estimation with the following factors:

$\chi_{S}$ The number of $i$ Pfam domain pairs observed in species $S$

$\theta_{S}$ The number of observed interactions in species $S$ that contain an $i$ Pfam domain pair

$\Theta_{S}$ The total number of observed interactions in species $S$

$\psi_{S}$ The number of proteins from species $S$ that are seen in an interaction screen

$\Psi_{S}$ The proteome size for species $S$

$\xi_{S}$ The number of Pfam domains observed in all protein of species $S$

$\Xi$ The total number of known Pfam domains

We denote the estimated number of $i$ Pfam domain pairs in species $S$ with $\hat{x}_{S}$. The formula we apply is

$$
\hat{x}_{S}=\chi_{S} \cdot \frac{\Theta S}{\theta_{S}} \cdot \frac{\Psi_{S}}{\psi_{S}}
$$

This means we scale the observed number of $i$ Pfam domain pairs to cover all observed interactions. We then use the relative proteome coverage to estimate the total number of $i$ Pfam domain pairs in all proteins.

Finally, we follow the argument of Aloy and Russel that the number of Pfam families seen in species $S$ indicates the fraction of the protein universe represented in the species. We therefore predict the total number of iPfam domain pairs $\hat{x}$ as

$$
\hat{x}=\hat{x}_{S} \cdot \frac{\Xi}{\xi_{S}}
$$

Both parameters and results of the calculation are shown in Table 3. The estimates for the total number of $i$ Pfam domain pairs ranges from 33813 to 120511 , with an average of 76918 .

\section{Discussion}

\section{iPfam coverage is low}

The coverage of $i$ Pfam on experimentally derived protein interactions is low. For S. cerevisiae, the species with the best mapped interactome, only $4.47 \%$ of the protein interactions contain an iPfam domain pair. Even in $H$. sapiens, where we suspect a positive bias due to the overrepresentation of disease-related proteins in both the PDB and protein interaction databases, $81 \%$ of protein interactions do not contain an $i$ Pfam domain pair. This reveals the limits of our understanding of the molecular structure of protein interactions.

Figure 1 also shows that a majority of protein interactions contains at least one pair of Pfam domains. While there is no structural information about putative interactions between these pairs, this fraction can already be analysed using statistical methods to identify putative domain interactions $[7,9,10]$. This in turn creates new targets for future structural genomics projects [30]. Prioritising these targets according to the number of covered experimental interactions could increase the coverage of databases like iPfam quickly.

We find, however, that $i$ Pfam domain pairs occur significantly more often in experimental interactions than 
Table 3: Prediction of total number of iPfam domain pairs

\begin{tabular}{|c|c|c|c|c|c|c|c|c|c|}
\hline Species & $\Theta_{S}$ & $\theta_{S}$ & $\Psi_{S}$ & $\psi_{\mathrm{s}}$ & $\chi_{s}$ & $\hat{x}_{S}$ & $\Xi$ & $\xi_{s}$ & $\hat{x}$ \\
\hline E. coli & $|28|$ & 211 & 4314 & 1163 & 347 & 7814 & 8957 & 2070 & 33813 \\
\hline S. cerevisiae & 45707 & 2045 & 5780 & 5359 & 835 & 20129 & 8957 & 2119 & 85085 \\
\hline C. elegans & 5310 & 221 & 22437 & 3022 & 197 & 35143 & 8957 & 2612 & | $205 \mid$ \\
\hline D. melanogaster & 21921 & $64 I$ & 16251 & 7023 & 266 & 21049 & 8957 & 2777 & 67893 \\
\hline H. sapiens & 24065 & 4577 & 38213 & 8179 & $122 \mid$ & 29994 & 8957 & 3476 & 77288 \\
\hline
\end{tabular}

$\Theta_{S}$ The total number of observed interactions in species $S$

$\theta_{S}$ The number of observed interactions in species $S$ that contain an iPfam domain pair

$\Psi_{S}$ The proteome size for species $S$

$\psi_{S}$ The number of proteins from species $S$ that are seen in an interaction screen

$\chi_{S}$ The number of iPfam domain pairs observed in species $S$

$\hat{x}_{S}$ The predicted total number of iPfam domain pairs in species $S$

$\Xi$ The total number of known Pfam domains

$\zeta_{s}$ The number of Pfam domains observed in all protein of species $S$

$\hat{x}$ The estimated total number of iPfam domains in all species

Prediction results are shown in bold font.

would be expected by chance. This requires that at least a subset of the iPfam domain pairs are reused in several experimental interactions.

\section{iPfam domain pairs can act as modules}

Despite the low overall coverage, $i$ Pfam domain pairs are found in more protein interactions than would be expected by chance (see Table 1). This statistical overrepresentation suggests that certain $i$ Pfam domain pairs constitute modules of molecular recognition which are reused in different protein interactions [2]. In fact, we find a characteristic power law distribution when we plot the histogram of experimental interactions per $i$ Pfam domain pair, see Figure 2. This underlines that a few promiscuous $i$ Pfam domain pairs are responsible for the majority of the $i$ Pfam coverage. These $i$ Pfam domain pairs are most likely to be reusable modules. In fact, we find the most frequent $i$ Pfam domain pairs to be recognition domains in signal transduction. Conversely, a large number of iPfam domain pairs are specific to a small number of protein interactions. This implies that recognition specificity amongst proteins is often achieved by maintaining an exclusive interacting domain pair. This could pose a problem for purely statistical approaches to infer domain interactions: if for many interfaces the real interacting domain pair will only occur once in an interactome, it will be hard to elucidate this on a statistical basis.

The concept of modularity of interacting domain pairs is furthermore supported by the positive correlation between the number of protein interactions an iPfam domain pair is seen in and the connectivity of the interacting proteins. We hypothesise that if during the course of evolution a protein is duplicated, it is likely to retain connections with other proteins which contain the same domain interaction modules. It is clear, however, that even though recognition domains are reused in various proteins, their specificity is bound to be controlled.

\section{Many domain-domain interfaces remain to be resolved}

We tried to estimate how many iPfam domain pairs exists in all interactomes. Our predictions lie almost an order of magnitude higher than the 10000 domain interaction types proposed by Aloy and Russel [2]. While all such estimates should be taken with caution, our results show that at best $10 \%$ of all structural domain pairs are represented in $i$ Pfam. The statistical approaches described in the introduction can only cover a small fraction of this domaininteraction space. Riley et al. for example report only 3005 interacting domain pairs which could be inferred from protein interactions [7]. Even under the assumption that many interactions involve short linear motifs, it seems likely that a large number of domain interactions remain to be resolved.

\section{iPfam domain pairs are conserved during evolution}

$i$ Pfam domain pairs are not only recurrent within the protein interaction network of one species. They also appear to be conserved between species. In a small set of protein structures from $S$. cerevisiae, it has been shown that interacting domain pairs are more conserved than non-interacting domain pairs [10]. Here, we call an iPfam domain pair conserved if there are protein interactions in two species which contain the same $i$ Pfam domain pair.

In a recent study [31], Gandhi et al. have assessed the conservation of protein interactions as the co-occurrence of orthologous interacting proteins. They found only 16 orthologous interacting protein pairs that were conserved in S. cerevisiae, C. elegans, D. melanogaster and H. sapiens. 


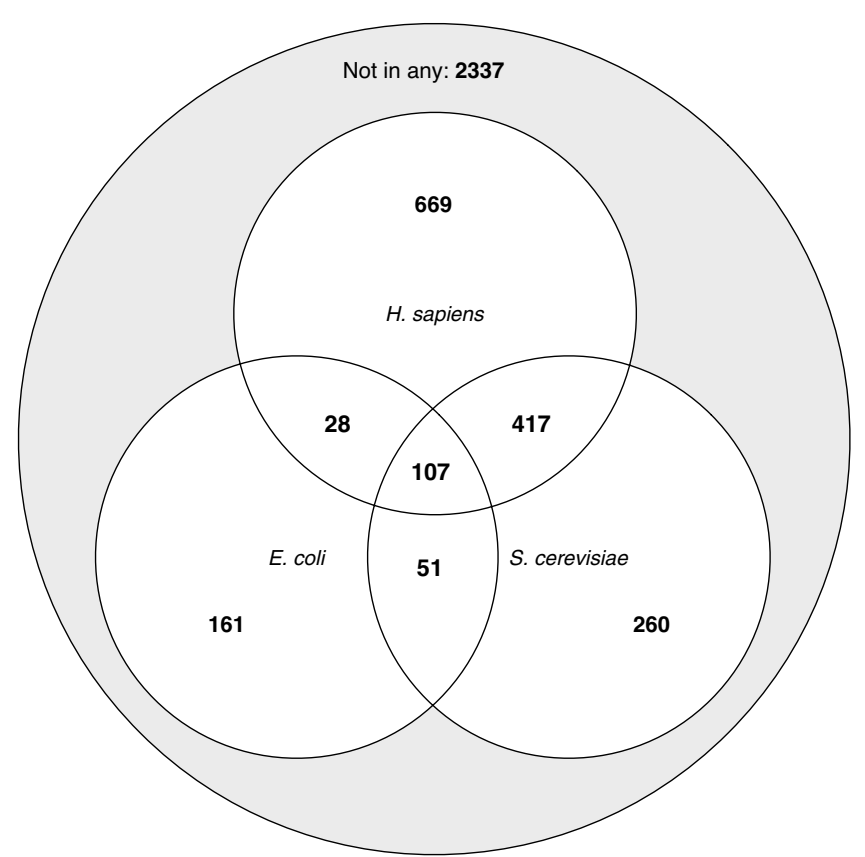

Figure 4

Venn diagramm showing the fractions of iPfam domain pairs found in the E. coli, S. cerevisiae and $H$. sapiens binary protein interaction sets. The three circles represent the iPfam domain pairs observed in the respective species. The overlaps denote co-observed iPfam domain pairs. The grey set in the background represents iPfam domain pairs not found in the three species.

Conversely, we find that $71 \mathrm{iPfam}$ domain pairs are conserved in the experimental interactions of these species. Even between a prokaryote like E. coli and the two eukaryotes $S$. cerevisiae and H. sapiens there is a considerable proportion of conserved iPfam domain pairs, to the extent that $53 \%$ of the $i$ Pfam domain pairs from E. coli are also observed in a eukaryote (Table 2). 107 domain pairs are shared between E. coli, S. cerevisiae and H. sapiens. These domains are predominantly related to transcription, translation and other basic essential cellular activities, which is in congruence with the findings of Gandhi et al..

Although the low overall $i$ Pfam coverage hampers the interpretation of our results, it looks as if there has been a diversification of domain interactions from E. coli to $H$. sapiens. While more than half of the $i$ Pfam domain pairs in E. coli have been retained throughout evolution, numerous new ones seem to have emerged in eukaryotic development. The significant positive correlation in the frequency of $i$ Pfam domain pairs conserved between $S$. cerevisiae and $H$. sapiens also suggests that the binding interfaces are more often kept or even reused rather than lost in the course of evolution. Conversely, this also raises the question of whether one could establish a comprehen-

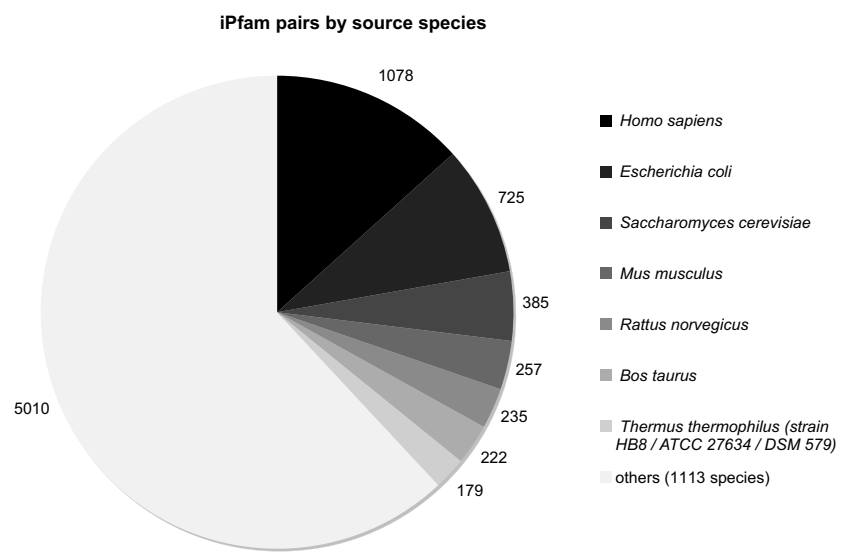

Figure 5

Species distribution of iPfam domain pairs. This pie chart shows how many iPfam domain pairs were found in PDB structures from each species. The total number is larger than the 4030 unique iPfam pairs in the database because an iPfam pair can be found in structures from several species.

sive set of domain interactions that were present in the last universal common ancestor.

\section{Conclusion}

In this study, we addressed the utility of current knowledge about structural domain interactions in order to interpret experimental protein interactions. Disappointingly, only a small fraction of all experimental interactions can be attributed to a known domain interaction. Within this subset of interactions, we nevertheless made several reassuring observations: structural domain pairs are enriched in experimental protein interactions. Some of the domain pairs seem to mediate a large number of protein interactions, thus acting as reusable connectors. This property is also conserved between species. Taken as a whole, this further underlines that solving structures of protein complexes should be an important focus for future structural genomics projects. Targeting the most frequent domain pairs would increase the coverage of databases such as $i$ Pfam, shedding more light onto the molecular mechanisms underpinning cellular networks.

\section{Methods}

\section{Protein interaction data}

The complete interaction sets from BioGRID [20], DIP [21], HPRD [22], IntAct [23] and MPact [24] were downloaded. A wide range of databases were used to cover as many distinct experimental data sets as possible. BioGRID for example contains a large manually curated set of protein interactions for S. cerevisiae [19]. Similarily, HPRD hosts a set of manually curated protein interactions for $H$. sapiens. IntAct on the other hand contains results from high-throughput screens and integrates data from other 
protein interaction databases as part of the IMEx collaboration. The MPact database combines the manually curated S. cerevisiae protein complexes data set formerly known as the MIPS complexes with other high-throughput interaction experiments data. Taken together, these databases represent most of the protein interactions currently stored in machine-accessible form.

Despite great efforts to unify access to protein interaction data [32], acquiring large data sets from diverse sources is still far from trivial and error prone. The PSI-MI XML data exchange format provided by the aforementioned databases was used to generate a local relational database of protein interactions. All entries were mapped to UniProt [33] by either relying on existing annotations from the source databases or by pair-wise sequence alignment to all UniProt proteins from the same species as the query protein. The direct sequence comparison was performed using pmatch, a very fast pairwise alignment algorithm developed by Richard Durbin (unpublished, source code available [34]).

\section{Species}

To allow cross-species comparisons, the data were split into five distinct species sets: E. coli, S. cerevisiae, C. elegans, $D$. melanogaster and $H$. sapiens. It should be noted that the proportion of proteins for which an interaction is known varies greatly between the species, see Table 1 . This might affect the results if there is a systematic bias on the composition of a protein interaction set.

To prevent bias from multiple alternative versions of the same protein, all interacting proteins were mapped to reference proteomes as defined by Integr8 [35], again using pmatch. An average of $\approx 16 \%$ of interaction entries were lost in the mapping process, either if no sequence was provided with the original entry or if no significant matching sequence could be found in Integr8. The total number of missing proteins will be lower, as several entries from different databases refer to the same sequence.

\section{iPfam}

The $i$ Pfam database is derived from protein structures deposited in the PDB. Regions in every protein structure that match a Pfam domain are scanned for interactions with residues in another Pfam domain. All such interacting domain pairs are stored in a database together with detailed information on the residues involved [13]. Every pair of Pfam families that are found to interact in a PDB structure are called an iPfam domain pair throughout the text. Single Pfam families that are part of an $i$ Pfam domain pair are then called iPfam domains. For example, in PDB entry $1 \mathrm{k} 9 \mathrm{a}$ the two $i$ Pfam domains SH2 (Pfam accession PF00017) and Pkinase_Tyr (PF07714) interact, therefore they form an $i$ Pfam domain pair. In this study, iPfam ver- sion 21 was employed, containing 2837 iPfam domains, forming $4030 i$ Pfam domain pairs. Figure 5 shows the species distribution of $i$ Pfam domain pairs. H. sapiens, $E$. coli and S. cerevisiae are clearly over-represented compared to the other 1113 species with less than 179 complex structures. Some iPfam domain pairs are seen to form interactions between distinct peptide chains in the structure (interchain), while others form an interaction between two distinct domains within the same chain (intrachain). In iPfam version 21, there are 3407 interchain and 1171 intrachain domain pairs, which means that 548 domain pairs mediate both inter- and intrachain interactions. In this analysis, both types of domain interactions were used equivalently, assuming that intrachain interactions can become interchain interactions and viceversa as a result of a gene-fission/fusion events.

\section{Filtering}

There are many types of experiments used to derive protein interactions, with different properties and error rates. For this analysis, solely the properties of physically interacting proteins is of interest. Therefore, only interactions between exactly two proteins per experiment were considered. That means all protein complex data that were derived by co-purification methods were removed, unless a particular experiment had identified exactly two binding partners. All genetic interactions were also removed.

\section{Random networks}

Randomised protein interaction networks with identical degree distributions were generated from the original filtered experimental interaction data for each species. In each randomisation step, a mapping is created that assigns every node a randomly chosen replacement node. In this way the edges of the network remain in place, while the nodes are shuffled randomly. It should be noted that the degree distribution per node is not maintained. Instead, this behaviour simulates a network with a high false positive rate.

\section{$P$ values}

$P$ values for observations $x$ were calculated as $P(X \geq x)=$ $f(x ; \mu, \sigma)$, where $f(x ; \mu, \sigma)$ is the probability density function of the normal distribution with mean $\mu$ and standard deviation $\sigma . \mu$ and $\sigma$ are estimated through the randomisation experiments. The density function thus provides the probability that a value less than or equal to $x$ is observed by chance, given the distribution estimated by a random resampling method. Where appropriate, the inverse probability $P(X>x)=1-f(x ; \mu, \sigma)$ was applied.

\section{Authors' contributions}

BSB wrote all software and carried out all the analyses. $A B$ contributed to the design and interpretation of the study. 


\section{Additional material}

\section{Additional file 1}

iPfam_pair_frequencies. En Excel file containing the frequencies of all iPfam domain pairs for all five species, i.e. the number of interactions that contain the respective iPfam domain pair.

Click here for file

[http://www.biomedcentral.com/content/supplementary/1471-

2105-8-259-S1.xls]

\section{Additional file 2}

iPfam_architectures. Two Excel sheets containing the frequencies of all iPfam domain architectures in $\mathrm{S}$. cerevisiae and $\mathrm{H}$. sapiens experimental interactions, i.e. the number of proteins that are composed of a certain set of iPfam domains.

Click here for file

[http://www.biomedcentral.com/content/supplementary/1471-

2105-8-259-S2.xls]

\section{Additional file 3}

iPfam pair_conservation. An Excel spreadsheet containing the 107 iPfam domain pairs conserved amongst $\mathrm{E}$. coli, S. cerevisiae and H. sapiens and their frequencies.

Click here for file

[http://www.biomedcentral.com/content/supplementary/1471-

2105-8-259-S3.xls]

\section{Additional file 4}

iPfam_frequencies. An Excel file containing the frequencies of each of the iPfam domains for all five species, i.e. the number of proteins containing the respective domain.

Click here for file

[http://www.biomedcentral.com/content/supplementary/1471-

2105-8-259-S4.xls]

\section{Additional file 5}

iPfam_inter-intrachain. An Excel file listing the number of interchain and intrachain interactions in all PDB structures for each iPfam domain pair.

Click here for file

[http://www.biomedcentral.com/content/supplementary/14712105-8-259-S5.xls]

\section{Acknowledgements}

The authors thank Robert Finn for valuable comments and suggestions. We are grateful for the numerous detailed comments and suggestions by the anonymous reviewers. BSB and $A B$ are funded by the Wellcome Trust.

\section{References}

I. Finn RD, Mistry J, Schuster-Bo?ckler B, Griffiths-Jones S, Hollich V, Lassmann T, Moxon S, Marshall M, Khanna A, Durbin R, Eddy SR, Sonnhammer EL, Bateman A: Pfam: clans, web tools and services. Nucleic Acids Res 2006:D247-25I.

2. Aloy P, Russell RB: Ten thousand interactions for the molecular biologist. Nat Biotechnol 2004, 22(10):1317-1321.

3. Itzhaki Z, Akiva E, Altuvia Y, Margalit H: Evolutionary conservation of domain-domain interactions. Genome Biol 2006, 7(I2):R 125

4. Ng SK, Zhang Z, Tan SH, Lin K: InterDom: a database of putative interacting protein domains for validating predicted protein interactions and complexes. Nucleic Acids Research 2003, 3I:25I-254.

5. Wuchty S, Almaas E: Evolutionary cores of domain co-occurrence networks. BMC Evol Biol 2005, 5:24.

6. Pagel $P$, Wong $P$, Frishman $D$ : A domain interaction map based on phylogenetic profiling. J Mol Biol 2004, 344(5): | 33 |- 1346.

7. Riley R, Lee C, Sabatti C, Eisenberg D: Inferring protein domain interactions from databases of interacting proteins. Genome Biol 2005, 6(1 0):R89.

8. Nye TMW, Berzuini C, Gilks WR, Babu MM, Teichmann SA: Statistical analysis of domains in interacting protein pairs. Bioinformatics 2005, 2 I(7):993-100I.

9. Lee $H$, Deng $M$, Sun $F$, Chen T: An integrated approach to the prediction of domain-domain interactions. BMC Bioinformatics 2006, 7:.

10. Jothi R, Cherukuri PF, Tasneem A, Przytycka TM: Co-evolutionary Analysis of Domains in Interacting Proteins Reveals Insights into Domain-Domain Interactions Mediating Protein-Protein Interactions. J Mol Biol 2006, 362(4):86I-875.

II. Park J, Lappe $M$, Teichmann SA: Mapping protein family interactions: intramolecular and intermolecular protein family interaction repertoires in the PDB and yeast. J Mol Biol 200I, 307(3):929-938.

12. Littler SJ, Hubbard SJ: Conservation of orientation and sequence in protein domain-domain interactions. J Mol Biol 2005, 345(5): 1265-1279.

13. Finn RD, Marshall M, Bateman A: iPfam: visualization of proteinprotein interactions in PDB at domain and amino acid resolutions. Bioinformatics 2005, 21 (3):4I0-4I 2.

14. Kouranov A, Xie L, de la Cruz J, Chen L, Westbrook J, Bourne PE, Berman HM: The RCSB PDB information portal for structural genomics. Nucleic Acids Res 2006:D302-305.

15. Peng K, Obradovic Z, Vucetic S: Exploring bias in the Protein Data Bank using contrast classifiers. Pac Symp Biocomput 2004:435-446.

16. Uetz P, Glot L, Cagney G, Mansfield TA, Judson RS, Knight JR, Lockshon D, Narayan V, Srinivasan M, Pochart P, Qureshi-Emlli A, Li Y, Godwin B, Conover D, Kalbfleisch T, Vijayadamodar G, Yang M, Johnston M, Fields S, Rothberg JM: A comprehensive analysis of protein-protein interactions in Saccharomyces cerevisiae. Nature 2000, 403(6770):623-627.

17. Ito T, Chiba T, Ozawa R, Yoshida M, Hattori M, Sakaki Y: A comprehensive two-hybrid analysis to explore the yeast protein interactome. Proc Natl Acad Sci USA 200I, 98(8):4569-4574.

18. Giot L, Bader JS, Brouwer C, Chaudhuri A, Kuang B, Li Y, Hao YL, Ooi CE, Godwin B, Vitols E, Vijayadamodar G, Pochart P, Machineni $\mathrm{H}$, Welsh M, Kong $Y$, Zerhusen B, Malcolm R, Varrone Z, Collis A, Minto M, Burgess S, McDaniel L, Stimpson E, Spriggs F, Williams J, Neurath K, loime N, Agee M, Voss E, Furtak K, Renzulli R, Aanensen N, Carrolla S, Bickelhaupt E, Lazovatsky Y, DaSilva A, Zhong J, Stanyon CA, Finley RL Jr, White KP, Braverman M, Jarvie T, Gold S, Leach M, Knight J, Shimkets RA, McKenna MP, Chant J, Rothberg JM: A Protein Interaction Map of Drosophila melanogaster. Science 2003, 302(565I): 1727-I736.

19. Reguly T, Breitkreutz A, Boucher L, Breitkreutz BJ, Hon GC, Myers $C L$, Parsons A, Friesen H, Oughtred R, Tong A, Stark C, Ho Y, Botstein D, Andrews B, Boone C, Troyanskya OG, Ideker T, Dolinski K, Batada NN, Tyers M: Comprehensive curation and analysis of global interaction networks in Saccharomyces cerevisiae. J Biol 2006, 5(4): II.

20. Stark C, Breitkreutz BJ, Reguly T, Boucher L, Breitkreutz A, Tyers M: BioGRID: a general repository for interaction datasets. Nucleic Acids Res 2006:D535-539.

21. Salwinski L, Miller CS, Smith AJ, Pettit FK, Bowie JU, Eisenberg D: The Database of Interacting Proteins: 2004 update. Nucleic Acids Res 2004:D449-45I.

22. Peri S, Navarro JD, Amanchy R, Kristiansen TZ, Jonnalagadda CK, Surendranath V, Niranjan V, Muthusamy B, Gandhi TKB, Gronborg M, lbarrola N, Deshpande N, Shanker K, Shivashankar HN, Rashmi BP, Ramya MA, Zhao Z, Chandrika KN, Padma N, Harsha HC, Yatish AJ, Kavitha MP, Menezes M, Choudhury DR, Suresh S, Ghosh N, Saravana R, Chandran S, Krishna S, Joy M, Anand SK, Madavan V, Joseph A, Wong GW, Schiemann WP, Constantinescu SN, Huang L, KhosraviFar R, Steen H, Tewari M, Ghaffari S, Blobe GC, Dang CV, Garcia JGN, Pevsner J, Jensen ON, Roepstorff P, Deshpande KS, Chinnaiyan AM, Hamosh A, Chakravarti A, Pandey A: Development of human 
protein reference database as an initial platform for approaching systems biology in humans. Genome Res 2003, I 3( I 0):2363-237I.

23. Hermjakob H, Montecchi-Palazzi L, Lewington C, Mudali S, Kerrien S, Orchard S, Vingron M, Roechert B, Roepstorff P, Valencia A, Margalit H, Armstrong J, Bairoch A, Cesareni G, Sherman D, Apweiler R: IntAct: An open source molecular interaction database. Nucleic Acids Res 2004:D452-455.

24. Güldener U, Münsterkötter $M$, Oesterheld $M$, Pagel P, Ruepp A, Mewes HW, Stümpflen V: MPact: the MIPS protein interaction resource on yeast. Nucleic Acids Res 2006:D436-44I.

25. von Mering C, Krause R, Snel B, Cornell M, Oliver SG, Fields S, Bork $P$ : Comparative assessment of large-scale data sets of protein-protein interactions. Nature 2002, 4I 7:399-403.

26. Grigoriev A: On the number of protein-protein interactions in the yeast proteome. Nucleic Acids Res 2003, 3 I ( I4):4I 57-4I6I.

27. Cusick ME, Klitgord N, Vidal M, Hill DE: Interactome: gateway into systems biology. Hum Mol Gen 2005, I4:RI7I-RI8I.

28. Aloy P, Ceulemans H, Stark A, Russell RB: The relationship between sequence and interaction divergence in proteins. J Mol Biol 2003, 332(5):989-998.

29. Gavin AC, Aloy P, Grandi P, Krause R, Boesche M, Marzioch M, Rau C, Jensen LJ, Bastuck S, Dumpelfeld B, Edelmann A, Heurtier MA, Hoffman V, Hoefert C, Klein K, Hudak M, Michon AM, Schelder M, Schirle M, Remor M, Rudi T, Hooper S, Bauer A, Bouwmeester T, Casari G, Drewes G, Neubauer G, Rick JM, Kuster B, Bork P, Russell RB, Superti-Furga G: Proteome survey reveals modularity of the yeast cell machinery. Nature 2006, 440(7084):63I-636.

30. Bravo J, Aloy P: Target selection for complex structural genomics. Curr Opin Struct Biol 2006, I 6(3):385-392.

31. Gandhi TKB, Zhong J, Mathivanan S, Karthick L, Chandrika KN, Mohan SS, Sharma S, Pinkert S, Nagaraju S, Periaswamy B, Mishra G, Nandakumar K, Shen B, Deshpande N, Nayak R, Sarker M, Boeke JD, Parmigiani G, Schultz J, Bader JS, Pandey A: Analysis of the human protein interactome and comparison with yeast, worm and fly interaction datasets. Nat Genet 2006, 38(3):285-293.

32. Hermjakob H, Montecchi-Palazzi L, Bader G, Wojcik J, Salwinski L, Ceol A, Moore S, Orchard S, Sarkans U, Von Mering C, Roechert B, Poux S, Jung E, Mersch H, Kersey P, Lappe M, Li Y, Zeng R, Rana D, Nikolski M, Husi H, Brun C, Shanker K, Grant SGN, Sander C, Bork P, Zhu W, Pandey A, Brazma A, Jacq B, Vidal M, Sherman D, Legrain P, Cesareni G, Xenarios I, Eisenberg D, Steipe B, Hogue C, Apweiler R: The HUPO PSI's Molecular Interaction format - A community standard for the representation of protein interaction data. Nat Biotechnol 2004, 22(2): I77-I83.

33. Wu CH, Apweiler R, Bairoch A, Natale DA, Barker WC, Boeckmann B, Ferro S, Gasteiger E, Huang H, Lopez R, Magrane M, Martin MJ, Mazumder R, O'Donovan C, Redaschi N, Suzek B: The Universal Protein Resource (UniProt): an expanding universe of protein information. Nucleic Acids Res 2006:DI87-I9I.

34. Sanger Institute CVS repository [http://cvs.sanger.ac.uk/cgi-bin/ viewcrs.cgi/rd-utils/]

35. Kersey P, Bower L, Morris L, Horne A, Petryszak R, Kanz C, Kanapin A, Das U, Michoud K, Phan I, Gattiker A, Kulikova T, Faruque N, Duggan K, Mclaren P, Reimholz B, Duret L, Penel S, Reuter I, Apweiler R: Integr8 and Genome Reviews: Integrated views of complete genomes and proteomes. Nucleic Acids Res 2005:D297-302.

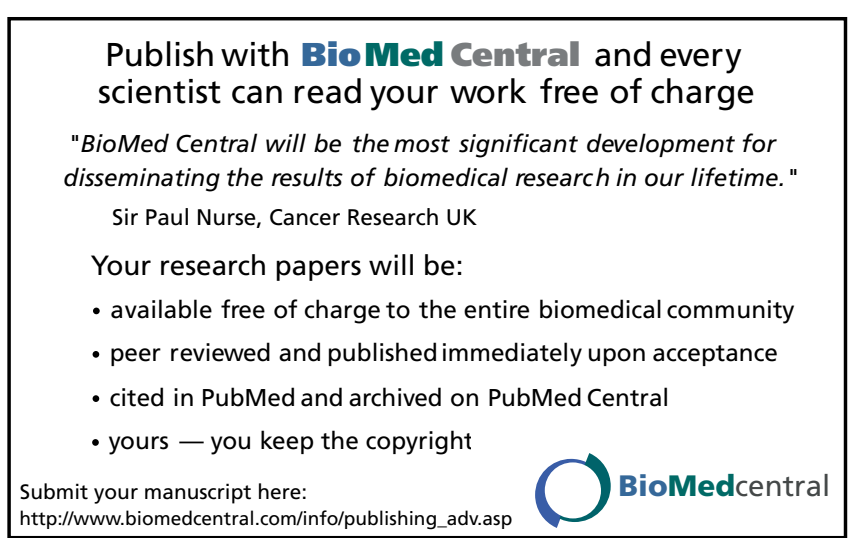

